\title{
Circuit
}

Musiques contemporaines

\section{La parodie dans Osten de Maurico Kagel}

\section{Andriy Talpash}

Volume 12, numéro 3, 2002

La route de soi

URI : https://id.erudit.org/iderudit/402004ar

DOI : https://doi.org/10.7202/402004ar

Aller au sommaire du numéro

Éditeur(s)

Les Presses de l'Université de Montréal

ISSN

1183-1693 (imprimé)

1488-9692 (numérique)

Découvrir la revue

Citer cet article

Talpash, A. (2002). La parodie dans Osten de Maurico Kagel. Circuit, 12(3), 57-62. https://doi.org/10.7202/402004ar

\section{Résumé de l'article}

Andriy Talpash s'interroge sur les mécanismes d'intégration qui régissent l'utilisation du folklore d'Europe de l'Est dans Osten, premier mouvement de l’oeuvre Die Stücke der Windrose de Kagel.
Ce document est protégé par la loi sur le droit d'auteur. L'utilisation des services d'Érudit (y compris la reproduction) est assujettie à sa politique d'utilisation que vous pouvez consulter en ligne.

https://apropos.erudit.org/fr/usagers/politique-dutilisation/ 


\title{
La parodie dans Osten de Maurico Kagel
}

\author{
Andriy Talpash \\ Traduit de l'anglais par Marc Hyland
}

Chaque écrivain invente ses propres précurseurs. Son ceuvre altère notre vision du passé, fout comme elle transformera l'avenir.

Jorge Luis Borges

(traduction libre)

Cette citation évoque bien l'immense gamme de sources d'inspiration et de théories dont dispose le compositeur d'aujourd'hui. Il a accès à une multitude de genres musicaux de toutes époques, auxquels il peut faire référence dans son travail. Ainsi, la disponibilité de ces ressources historiques les posent non seulement comme modèles à dépasser, mais aussi comme matériaux potentiels pour la composition. Le mouvement Osten, tiré de l'œuvre Die Stücke der Windrose (1988-1994), de Mauricio Kagel, relève de cette dernière possibilité. Le traitement et la déformation, par Kagel, de musique folklorique déjà existante illustre sa "vision altérée du passé". Les références que Kagel fait à des matériaux préexistants dans son œuvre posent une série d'interrogations à l'auditeur qui sait les reconnaître: Dans quel projet théorique ses références à d'autres textes s'insèrentelles? Comment pourraiton caractériser l'emploi de la référence chez Kagel : parodie? allusion? intertextualité stylistique? citation? une forme de pastiche? Eł pourquoi Kagel faitil seulement référence à un autre style? Une analyse succincte de Osten nous permettra de conclure que c'est la notion de parodie qui fonde et nomme le plus justement ce mouvement.

Composé en 1988-1989, Osten constitue le premier des cinq mouvements de Die Stücke der Windrose. Pour y réaliser son interprétation d'une "scène" particulière, Kagel intègre et fait référence à plusieurs éléments de langage typiques du folklore d'Europe de l'Est, notamment la forme, le rythme, les cellules motiviques, les échelles, les textures et les timbres. Cette "scène" représente en quelque sorte un scénario: le compositeur est passager de troisième classe dans un train qui défile à travers l'Europe de l'Est. Kagel en a fourni la description suivante :

Au nombre des autres voyageurs figure un groupe de musiciens qui semblent tout droit sortis d'un vieil album photo. lls commencent à jouer pour moi. Les ondulations 
du paysage appellent des styles d'interprétations appropriés : les fragments mélodiques et les rythmes typiques défilent encore plus rapidement que ne le font les villages qui glissent sous nos yeux'.

En observant la structure formelle de Osten, on remarque que certaines sections se répètent. Celles-ci, ainsi que les sections "um-pa " et les sections improvisées et ornées qui se trouvent mises en contraste, sont extrêmement répandues dans le folklore esteuropéen. De façon générale, la forme de Osten est très proche de cette tradition, à celte différence près que Kagel y déforme le rythme "um-pa" traditionnel de la polka : selon la convention, le "um » coincide avec le temps fort, tandis que le "pa» tombe sur le temps faible. Kagel procède pour sa part à de nombreuses inversions de ce rythme, mais la référence au modèle n'en reste pas moins évidente.

L'usage très fréquent de secondes augmentées et de tritons dans la mélodie, entendus essentiellement à la clarinette tout au long de Osten, crée bien une ambivalence majeur et mineur, mais aussi un mode lydien altéré. Cette prédominance accordée aux secondes et aux tritons est l'une des principales caractéristiques mélodique et motivique du folklore est-européen.

l'instrumentation choisie par Kagel pour Osten évoque à son tour celle des groupes traditionnels de l'Europe de l'Est : clarinette, piano, harmonium, cordes $2 / 1 / 1 / 1$, et percussions. Cette combinaison instrumentale produit une texture à la fois métallique, froide et sèche, qui se trouve rehaussée par l'intégration que fait Kagel des sons harmoniques et sul ponticello aux cordes, ainsi que par l'usage de I'harmonium et des instruments percussifs métalliques: tambourin, cymbales, triangles et grelots. L'instrumentation et l'orchestration de Kagel font clairement référence aux sonorités percussives et métalliques du dulcimer ou cymbalum, un instrument populaire dans plusieurs régions d'Europe de l'Est.

L'influence de la musique juive klezmer est également présente dans Osten. Kagel est né de parents russes juifs, émigrés en Argentine autour de 1900. Qu'il pratique ou non la religion juive, les sonorités créées dans Osten ont des liens étroits avec celles du folklore yiddish.

Après ce survol rapide de Osten, nous devons considérer la question de la référence à d'autres genres musicaux dans l'œeuvre de Kagel. Nous nous sommes volontairement abstenu jusqu'ici d'employer le terme de parodie pour qualifier la technique compositionnelle de Kagel - celle où il fait des références à d'autres styles musicaux. Pourquoi? Parce que ce mot est chargé de multiples sens, nuances et sous-entendus, et peut en cela prêter à confusion lorsqu'il est accolé à $n$ 'importe quelle œuvre. Une musique qualifiée de parodique est généralement perçue comme porteuse d'une dimension humoristique :

[...] produits qui imitent de façon partielle ou intégrale la forme d'un texte connu, ou les degrés secondaires de ses apparences, manières et coutumes, événements ou
1. Mauricio Kagel, livret de Mauricio Kagel 5. Stücke der Windrose: Osten, Süden, Nordosten, Nordwesten, Südosten; Phantasiestück. Reinbert de Leeuw et le Schönberg Ensemble. Disques Montaigne MO 782017. 
personnes. Cette imitation, à première vue fidèle, se trouve déformée par un humour volontaire ef caractéristique ${ }^{2}$.

Le dictionnaire Oxford offre une autre définition de la parodie :

Une composition en prose ou en vers où les éléments de style et de pensée typiques à un auteur ou à un type d'auteurs sont imités de façon à les rendre ridicules, plus spécifiquement en étant appliqués à des sujets totalement inappropriés; l'imitation plus ou moins fidèle d'une œuvre, conçue pour produire un effet de ridicule.

(traduction libre)

À la lumière de ces deux définitions, il semble inapproprié de qualifier Osten de parodie. II n'y a pas de dimension humoristique dans la "distorsion" opérée ici par Kagel du folklore esteuropéen, et encore moins d'humour, croyons-nous, si l'intention de Kagel était d'intégrer les sonorités du folklore yiddish.

Cependant, le concept de parodie est souvent utilisé et invoqué à tort dans la littérature et la musique. Au cours des derniers siècles, on a associé ce terme aux multiples acceptions propres à la satire. Satire et parodie constituent des notions distinctes, mais la dimension de dénigration comique de la satire a été attribuée à la parodie, ce qui est une erreur.

Le mot parodie tire sa source étymologique du substantif grec parodia, qui veut dire "contre-chant». Le préfixe para signifie contraste ou opposition entre des textes. C'est ici, dans cette situation où des textes s'opposent, que les théoriciens introduisent fréquemment une dimension de ridicule. Mais le préfixe para signifie aussi "qutour", suggérant alors une proximité et un accord entre des textes. C'est pourquoi l'humour n'a pas sa place dans la parodie. Celle-ci, "dans l'ironie de sa transcontextualisation et de son inversion, consiste bien plutôt en une répétition altérée ${ }^{3}$.

Contrairement à ce qu'on a pu croire, la parodie et la satire peuvent coexister sans pour autant que la satire soit inhérente à la parodie. Plus précisément, "la satire adopte fréquemment le mode de la parodie, se faisant ainsi le véhicule de sa moquerie des travers et de la bêtise de l'homme, dans une perspective corrective ${ }^{4}$. D'une cerłaine façon, la parodie consiste en une simple opposition de textes, sans exigences sémantiques, tandis que la satire exige une variation du niveau sémantique dans l'intention. Ainsi, on se retrouve avec le modèle suivant : parodie $\rightarrow$ un signifiant; satire $\rightarrow$ un signifiant et deux signifiés. Dans la satire, "le refus systématique d'une univocité sémantique correspond au refus d'une unitextualité structurelle dans la parodie ${ }^{5}$.

Qualifier Osten de parodique, à la lumière de ces définitions, équivaudrait à faire de la parodie la technique compositionnelle privilégiée de Kagel. Celvi-ci a besoin de ces relations à d'autres types de musiques, sur le plan intrinsèque ou extrinsèque; la parodie n'est rien de plus qu'une technique pour le compositeur.
2. Paul Lehmann, Die Parodie im Mittelalter, Stutfgart, Hiersemann, 1963, p. 16.

3. Linda Hutcheon, A Theory of Parody, New York, NY, Methuen, Inc., 1985. p. 32.

4. Hutcheon, p. 54

5. Hbid., p. 54. 
Il est impossible de composer sans mettre de l'avant certains liens ou rapports. De fait, la composition est à la fois un programme ou un plan au cours duquel un choix est fait en faveur d'une musique spécifique, excluant du coup toutes les autres. II ne pouvait y avoir d'autre voie que cette confrontation soutenue avec le passé ${ }^{6}$

Une autre dimension fondamentale de cette caractérisation de Osten en tant que parodie consiste dans la perception/réception d'un texte parodique. Le rôle de l'auditeur est primordial dans ce processus " $\mathrm{d}^{\prime}$ encodage et de décodage ${ }^{7}$ " du parodique. Pour une lecture parodique d'un texte, l'auditeur doit reconnaitre les éléments extratextuels mis en jeu. "L'identité structurelle du texte comme parodie dépend alors de la coïncidence critique, du décodage (reconnaissance et interprétation) et de l'encodage $^{8}$ "Selon Linda Hutcheon, "la parodie prend son plein essor pendant les périodes de complexité culturelle, qui permettent aux parodistes de s'en remettre à l'érudition du lecteur (spectateur, auditeur) de la parodie" ". "Les promenades inférentielles ne sont pas que des artifices [...] mais sont bien générées par des structures discursives et anticipées par la stratégie textuelle globale comme éléments essentiels de la structure de la fabula ${ }^{10}$ ". Deux aspects de la parodie s'imposent : le premier consiste à reconnaitre que le texte constitue une parodie, tandis que le second est de reconnaître le texte d'origine qui se trouve parodié. Considéré sous cet angle, le lecteur de la parodie joue un rôle actif ${ }^{1}$, dans la création du texte parodique.

Toutefois, "si le décodeur est incapable de remarquer ou d'identifier une allusion ou une citation, il ou elle opère alors une intégration au premier degré de cette matière, adaptant alors son interprétation au contexte global proposé par l'œuvre ${ }^{12}$ ", ratant potentiellement du même coup le véritable enjeu de l'œuvre. Étonnamment, cette "intégration au premier degré" est ce à quoi aspire le plus souvent Kagel :

[...] arriver, par une multiplication minutieuse de matériau en « circuit fermé », à constituer un montage extrêmement serré de pièces plus ou moins connues - sans l'addition d'un seul matériau étranger. L'attention de l'auditeur pourra ainsi être concentrée sur la substance musicale du contexte, au lieu d'être constamment distraite par ces moments anecdotiques de reconnaissance ${ }^{13}$.

Kagel sousentend donc que la parodie ne constitue pas une stratégie dans sa pratique de la composition. Mais il reste impossible de nier l'existence de liens ou d'influences du folklore est-européen dans Osten. Ce mouvement dénote très clairement une influence de ce folklore musical, sous-entendue dans le titre Osten lau sens géographique de "est»|, et une dimension programmatique. Par conséquent, dans le cas qui nous occupe, un point demande réflexion : quelle est l'intention du compositeur/auteur, et quel rôle joue cette intention lorsque la parodie est utilisée comme technique formelle dans la composition? Pourquoi Kagel faitil une parodie du folklore est-européen dans Osten? On pourrait avancer que Kagel parodie une musique déjà existante pour maintenir une distance entre lui et l'œuvre. Kagel se sert de la parodie "comme d'une méthode pour insérer une continuité lout en pré-
6. Andrew D. McCredie

* Kagel..../1991 *, Notes, vol. 51, sept. 1994, p. 163

7. Hutcheon, p. 34 .

8. Ibid., p. 34

9. Ibid., p. 19

10. Umberto Eco, The Role of the Reader. Bloomington, Ind, Indiana University Press, 1979, p. 32.

11. Hutcheon, p. 93

12. Ibid., p. 34

13. Richard Kostalenetz, "Polyartist" Opera News, vol. 53, sept. 1988, p. 30. 
servant une distance critique ${ }^{14} 》$. Bien que Osten ne présente pas de citation directe du folklore musical d'Europe de l'Est, Kagel maintient effectivement une distance entre lui et l'œuvre par l'interprétation qu'il en offre. Peut-être parce que "l'écart ironique rendu possible par la parodie a donné un souffle de liberté à la technique de l'imitation ${ }^{15}$ ". Ian Pace croit que chez Kagel,

[...] la création d'un jamaisencore-entendu à partir de distorsions, modifications, juxtapositions ou contextualisations originales de fragments ou de matériaux déjà existants, pourraif être considérée comme une réponse artisanale ò la conception bourgeoise qui fait de l'artiste une figure solitaire, voire solipsiste, un être imperméable aux goûts populaires, et jugé digne dépositaire d'une reconnaissance lorsqu'il a trouvé sa "propre voix". Ceci étant dit, l'usage inconsidéré de clichés expressifs peut constituer une manœuvre opportuniste pour combler la nostalgie du public tout en apaisant sa peur de la nouveautét ${ }^{\text {to }}$.

La dernière partie de cette citation soulève une autre interrogation. Peut-être estce à l'intention du public que Kagel parodie des matériaux déjà existants, pour faciliter leur reconnaissance et l'appréciation de "simples mélodies". Hutcheon croit que la parodie est "un des moyens qui a permis aux artistes modernes de régler leur contentieux avec le poids du passé ${ }^{17}$ "; le passé devrait être un "perpétuel présent ${ }^{18}$ ". II n'en reste pas moins que cette question de l'intention résiste à toute interprétation définitive, et des spéculations plus avancées ne mèneraient à rien de plus.

Quant au rôle de l'intention, Stanley Fish affirme que "nous ne pouvons comprendre un texte indépendamment d'une intention, i.e. de l'hypothèse que les signes ou les sons livrés par le texte ont été produits par l'intention d'un être, un être faisant partie d'un ensemble en relation avec lequel il a un rôle ou un point de vue ${ }^{19}$ ". Cette théorie semble beaucoup trop extrême pour s'appliquer à Osten. Pourtant, même si nous ne connaissons pas toute la mesure de l'intention de Kagel lorsqu'il utilise une technique de composition ou une autre dans Osten, chaque lecture de l'œuvre provoquera néanmoins un jugement esthétique.

En conclusion, it nous apparaît que Osten est un exemple patent de parodie dénuée de cette dimension satirique et grotesque qu'on lui a souvent attribuée jusqu'ici dans l'histoire. II nous semble également qu'une interprétation juste de Osten doit mettre en valeur son aspect parodique puisqu'il s'agit du principal moteur compositionnel de l'œuvre. Si pour être réussie, la parodie exige la participation de l'auditeur, notamment en ce qui a trait à une connaissance des textes et des objets qui s'y trouvent parodiés, on est en droit de s'interroger sur la perception de l'œuvre par l'auditeur. Kagel était bien sûr conscient qu'il parodiait des éléments de folklore musical d'Europe de l'Est, mais l'intention précise à l'origine de son geste risque fort de ne jamais être entièrement reconnu. Kagel partagerait sans doute le point de vue de Karlheinz Stockhausen : "Entendre une matière musicale familière, passée, déjà formée, avec des oreilles vierges, pour la pénétrer et la métamorphoser avec une
14. Hutcheon, p. 20.

15. Hbid., p. 35

16. lan Pace, "Music of the Absurd? Thoughts on recent Kagel», Tempo: A Quarterly Review of Modern Music, 11997 env.l, p. 34.

17. Hutcheon, p. 29.

18. Ibid., p. 42.

19. Stanley Fish, "Working on the Chain Gang: Interpretation in the Law and literary Criticism 》, Critical Inquiry, 9, 1982, p. 213. 
conscience musicale de notre époque ${ }^{20} "$. Pour tout dire, Kagel n'a fail que "remanier des formes connues pour leur donner plus d'impact et de gravité ${ }^{21}$ ».

\section{Bibliographie}

DANE, J. A. (1988), Parody: Critical Concepts versus Literary Practices: Aristophanes to Sterne. Norman, Oklahoma, University of Oklahoma Press.

DANE, J. A. (1980), "Parody and Satire: A Theoretical Model », Genre, 13, p. 145159.

DUFFALO, R. (1989), Trackings: Composers Speak with Richard Duffalo. New York, Oxford University Press.

GROUT, D. J. (1988), A History of Western Music, 4th Ed. New York, New York, W.W. Norton \& Company, Inc.

HÄUSLER, J. (1980), "Mauricio Kagel " The New Grove Dictionary of Music and Musicians, sous la d. de Stanley Sadie, vol. IX, p. 766-769. London, Macmillan Publishers Lid.

HUTCHEON, L. (1985), A Theory of Parody. New York, Methuen, Inc.

KAGEL, M. (1990), Die Stücke der Windrose: für Salonorchester: Osten, partition. Frankfurt, New York, H. Litolff's Verlag, C. F. Peters.

KAGEL, M. (1994), Die Stücke der Windrose: Osten, Süden, Nordosten, Norwesten, Südosten; Phantasiestück. Reinbert de Leeuw et le Schöenberg Ensemble. Montaigne, MO 782017.

KOSTALENETZ, R. (1988), « Polyartist», Opera News, vol. 53 p. 28-30.

MCCREDIE, A. D. (1994), "Kagel.../1991", Notes vol. 51, p. 162-167.

MORRIS, C. (1938), Foundations of the Theory of Signs, I. Chicago, Illinois, University of Chicago Press.

NATTIEZ, J.J. (1990), Music and Discourse: Toward a Semiology of Music. Princeton, New Jersey, Princeton University Press.

PACE, I. (1997), "Music of the Absurd? Thoughts on Recent Kagel ", Tempo: A Quarterly Review of Modern Music. p. 29-34.

RABINOWITZ, P. J. (1981), "Fictional Music: Toward a Theory of Listening "Theories of Reading, looking and listening, sous la d. de Harry R. Garvin, p. 193-208. Lewisburg, Pennsylvania, Bucknell University Press.

RABINOWITZ, P. J. (1980), "What's Hecuba to Us?" : The Audience's Experience of Literary Borrowing ", The Reader in the Text: Essays on Audience and Interpretation, sous la d. de Susan R. Suleiman \& Inge Crosman, p. 241-263. Princeton, New Jersey, Princeton University Press.
20. Donald J. Grout (1988), A History of Western Music, $4^{\mathrm{e}}$ ed., New York, NY, W.W. Norton \& Company, Inc., p. 874. 21. Rose Rosemary Freeman (1963), «Parody as a Literary Form: George Herbert and Wilfred Owen», Essays in Criticism, 13, p. 307. 